\title{
Fiber vibrometer with three-phase fringe-analysis
}

\author{
Mark Johnson ${ }^{1}$ and Yves Delaporte \\ Optoelectronics Research Centre, University of Southampton, Southampton SO9 5NH, UK
}

Received 11 March 1993

\begin{abstract}
A fiber-remoted, non-contact, optical surface-vibrometer which uses no source wavelength modulation is reported. Three-phase fringe sampling using a $3 \times 3$ fiber coupler and processing using a digital processor allow automatic multi-fringe unwrapping, subfringe interpolation and insensitivity to interferogram intensity and contrast variations. A noise-equivalent sensitivity of $50 \mu \mathrm{fringe/}$ $\sqrt{\mathrm{Hz}}$ is demonstrated.
\end{abstract}

\section{Introduction}

Optical interferometers are increasingly being used in mechanical metrology in preference to other techniques [1]. They function by beating an optical reference-wave with a signal-wave reflected from the object under investigation, forming an interferogram. The non-contact nature of the measurement gives optical interferometry significant advantages over, for example, piezoelectric accelerometers, including minimal loading of the surface under measurement, very high spatial resolution, and high measurement bandwidths. In addition, conjunction with optical fibers gives the ability to measure in inaccessible locations with a passive optical head. Lastly, new semiconductor and rare-earth-doped fiber sources promise reduced system complexity and cost. and operation at a wider range of wavelengths.

In practice, however, many demonstrated systems are complex. because of the need for fringe processing to remove directional ambiguity and sensitivity variation inherent in the periodic interference process. Many published and commercial systems use phase- or frequency-modulation (heterodyne) techniques to circumvent these problems. Where the source is a gas-laser an expensive Bragg-cell frequency shifter is required for this. Direct frequencymodulation of diode-laser sources is possible, but

1 Present address: North West Water Group, Warrington, Cheshire WA5 3LW. UK. only by introducing new problems of simultaneous intensity-modulation and laser instability via modehopping. In the scheme described here no source wavelength modulation is necessary. Instead, static measurements of interferogram intensity are made at several different optical phases. The resultant system is much simpler, uses primarily digital processing techniques, and operates the source in an optimum environment of constant temperature and drive conditions. In principle, any source of adequate temporal coherence may be used, without regard for the requirement for direct-modulation.

\section{Experimental configuration}

Figure 1 shows the optical configuration. Light from a $40 \mathrm{~mW} 841 \mathrm{~nm}$ wavelength semiconductor laser (Sharp LT015) was coupled into a length of non-polarization-maintaining singlemode fiber using two lenses $(f=3.6 \mathrm{~mm}, \mathrm{NA}=0.45 \mathrm{Nippon}$ Sheet

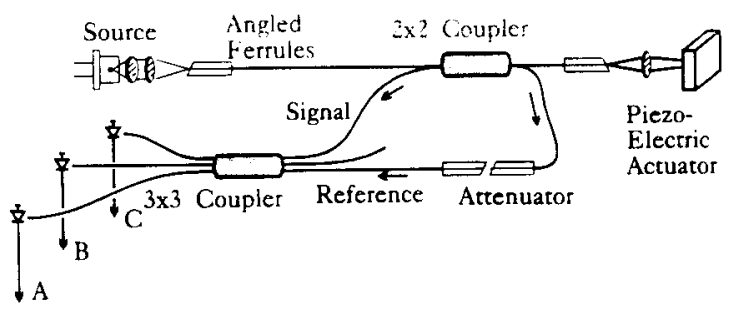

Fig. 1. Three-phase vibrometer optical configuration. 
Glass type SPL graded index lens and $f=10 \mathrm{~mm}$ Grun Optik GmbH. achromat). All fiber ends were angle-polished at $15^{\circ}$ to reduce back-reflection of light into the laser cavity. Overall coupling efficiency was $\sim 42 \%$.

At the distal fiber end the light was collected using an $f=30 \mathrm{~mm}$ Steinheil achromat (Melles Griot 01 LAT 15 ) and focused onto the vibrating surface of a $20 \mathrm{~mm}$ diameter piezo-electric buzzer, coated in retroreflecting paint. By driving the buzzer electrically, displacements up to a few $\mu \mathrm{m}$ were possible. About $10^{-5}$ of the incident light was recollected by the emitting fiber.

$100 \mathrm{~mm}$ from the emitting fiber end approximately half of the light was split off in a $2 \times 2$ fiber coupler to serve as a reference wave, after attenuation in an in-line fiber attenuator formed using a fixed air-gap. The remaining port of the $2 \times 2$ coupler separated half of the reflected signal wave. To form the interferogram, object and reference waves were mixed in a second, $3 \times 3$ fiber-coupler. Only two input ports of this coupler were used.

\section{Signal processing}

The interferogram to be measured has the sinusoidal form $l=M+R \cos \phi$, being described by three unknowns, namely the mean detected intensity $M$, the contrast $K=R / M$, and the phase $\phi$. In order to determine $\phi$, independent of variations in the other quantities. at least three independent measurements of the fringe pattern must be made. A pair of (quadrature ) measurements spaced by $\phi=\pi / 2$ is not adequate unless variations in contrast and mean intensity are small, or can be calibrated out by sweeping the phase. as in heterodyne techniques. Through the use of the $3 \times 3$ coupler, whose outputs are mutually phase shifted by approximately $2 \pi / 3$ radians. three independent samples of the interferogram were made. This configuration is much more convenient, more stable. and optically efficient than a system manipulating fringes in a bulk Michelson interferometer [2].

Normalization of received contrast level and phase determination using analogue circuitry via the relation $\phi=\tan ^{-1}(Q / I)$ is commonly used, but superior performance can be obtained by converting at an early stage to the digital domain, afterwards translating digital intensity values to phase values in EPROM look-up tables. This elegant approach was developed by Mertz [3,4] for use in astronomical instrumentation. The parallel digitization technique demonstrated in that work is ideally suited to this application (fig.2).

The three optical samples were detected in identical transimpedance amplifier receivers with a transimpedance of $100 \mathrm{M} \Omega$. This high value was dictated by the low level $(\sim \mathrm{nW})$ of received power. Using a dual operational-amplifier design it was possible to achieve a system bandwidth of $>80 \mathrm{kHz}$ with an unbiased $1 \mathrm{~mm}^{2}$ detector of $20 \mathrm{pF}$ capacitance.

The three electrical signals were then fed in parallel to a bank of six 8-bit flash analog to digital (ADC) converters (Micro Power Systems Inc. MPS8780). Each ADC was responsible for digitizing $2 \pi / 6$ of a fringe, namely the approximately linear branch in the most rapidly varying portion of the sinusoidal fringe pattern. Note the permutation of the three signals $\mathrm{A}, \mathrm{B}, \mathrm{C}$ amongst the high-reference, low-reference and true inputs of the ADCs. Overflow flags on three of the ADCs encoded a chip-select bit

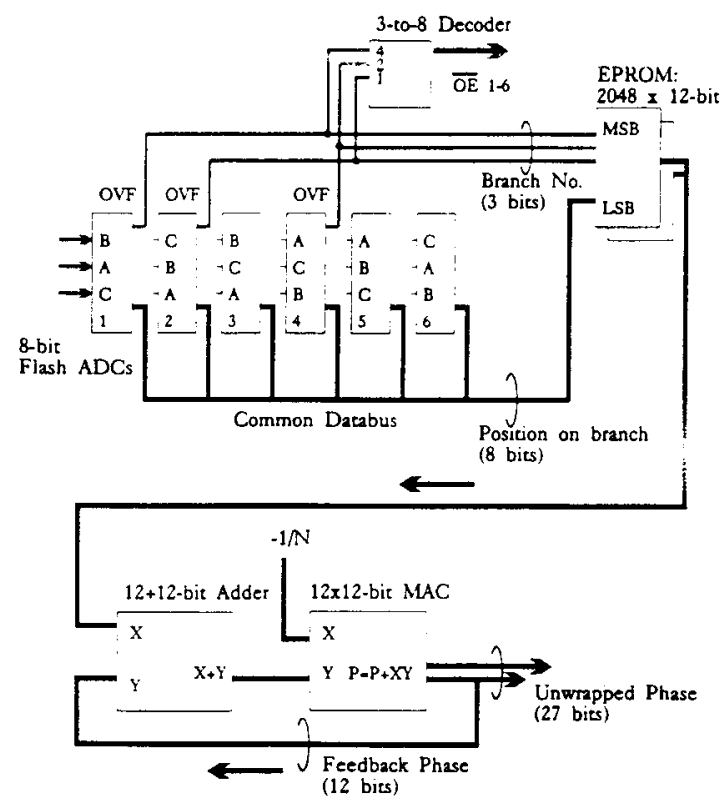

Fig.2. Digital processor which performs phase measurement in a parallel-connected bank of ADCs. and low-pass filtering with a multiplier accumulator chip (MAC). 
that was used to switch valid data onto a common data-bus. As the optical phase was varied, the six ADCs encoded intensity into $6 \times 256=1536$ resolved points per cycle. A pair of fast EPROMs (Cypress Semiconductor Corp. CY7C291) coded with the remanent sinusoidal nonlinearity supplied the 1536 per fringe phase values to 12 -bit precision.

This technique [3] of digitally normalizing each intensity sample to a span given by the other two phase samples removed all effects of absolute intensity and contrast variations. As long as the three samples exhibit the same mean intensity and contrast values, and suffer from zero intensity offsets, hen a reduction in intensity or contrast does not change the measured phase, but only the signal-tonoise ratio $(\mathrm{S} / \mathrm{N})$.

While the basic digitization-limited resolution was adequate for a machine-iool application, a resolution much higher than $(2 \times 1536)$ was necessary for surface vibration measurements. This was achieved by low-pass filtering, performed as in ref. [3] using a digital infinite-impulse-response (IIR) filter designed around an adder and 12 $\times 12$-bit multiplier-accumulator chip (MAC). Filtering bandwidth and resolution-enhancement could be chosen by setting the number $N$ (division input) to the MAC in the range $2^{0}$ to $2^{10}$. $N$ determines the number of clock cycles over which any input phase-change is averaged by the IIR filter; the effective sampling rate is therefore clock-rate $/ N$. The MAC also performed fringe-counting to 16 fringes directly, and to arbitrarily large number of whole fringes using external circuitry.

\section{Performance}

The 25-bit dynamic range capable of the MAC s , tem (11-bit sampling plus 10-bit filtering plus 4-bit fringe counting) cannot be resolved on an oscilloscope display, so an 8-bit digital-to-analog converter (DAC) was used to display a selected 8-bit segment from the full phase output. By moving the location of the 8 bits along the full output, full-scale analog deflections (FSD) up to 16 fringes could be displayed, or down to 1/1024th fringe. Figure 3 shows one channel of the raw interferometer output (a) and demodulated motion (b) for a multi-fringe $(\sim 8 \lambda)$

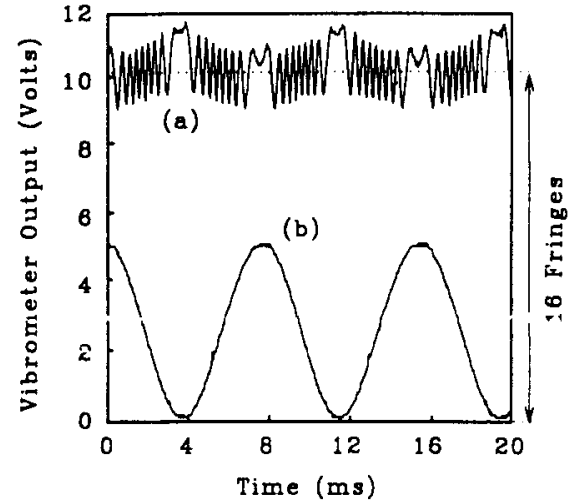

Fig.3. Detected interferogram and demodulated object position for an $\sim 8 i$ peak-to-peak sinusoidal motion.

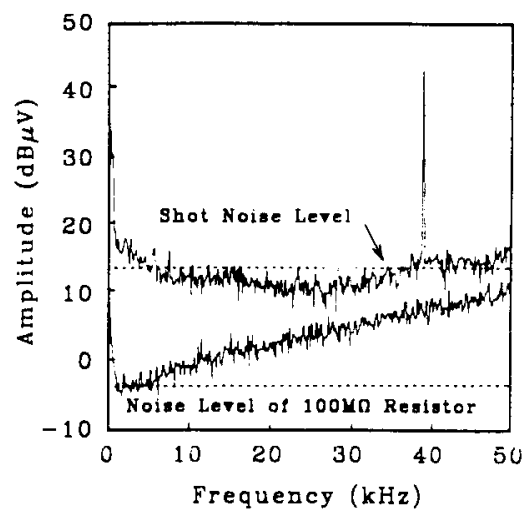

Fig.4. Noise spectrum of unilluminated photoreceiver (lower trace) and vibrometer output (upper trace) with an object motion of $4 \pi \mathrm{mrad}$ peak-to-peak.

motion. Here the sampling rate was $7.5 \mathrm{MHz}$, reduced to $59 \mathrm{kHz}$ with an $N=128$ filtering divisor. At this high amplitude some intensity modulation due to transverse movement on the retroreflector beads is visible. However, the demodulated output is undistorted. showing the robustness of the three-phase system to such effects.

At a source power level of $10 \mathrm{~mW}, 2 \mathrm{~mW}$ was incident on the target. The received power level using retroreflective tapes was very variable, but typically an object signal of $2 R \equiv 40 \mathrm{nW}$ was available. A peakto-peak (pk-pk) object motion of $>420 \mathrm{~nm}$ ( 1 fringe pk-pk motion) would therefore give $2 \mathrm{~V}$ output at the receivers. System bandwidth was set at $80 \mathrm{kHz}$. Figure 4 shows the interferometer noise spectrum by 
comparison with a $4 \pi$ mrad object phase excursion at $40 \mathrm{kHz}$. Dotted lines give the shot noise levels due to detector dark current and a $40 \mathrm{nW}$ received power level. The apparent sub-shot noise level at mid frequencies is due to $\sim 3 \mathrm{~dB}$ variations in receiver frequency response reducing detected output there also. The noise level corresponds to a minimum detectable phase in $1 \mathrm{~Hz}$ bandwidth of $\sim 12 \mu \mathrm{fringe}$; we speciîy the instrument at $50 \overline{\mathrm{f}}$ fringe $/ \sqrt{\mathrm{Hz}}$ to allow for reflectivity variations of the retroreflective coatings.

\section{Summary}

In contrast to heterodyne, modulated diode-laser based vibrometers, the multi-phase static scheme described here places the source in an improved environment, namely optically-isolated from the fiber delivery system, at constant temperature, and driven from a low-noise power-control circuit. As no ac phase-modulation is required of the source, it can equally well be a gas laser, doped fiber-laser. or broadband emitter for short path-difference systems. A disadvantage of the present scheme is that the fibers in the head are not insensitive to phase disturbances, and so the phase will tend to drift over time. However, with careful encapsulation drift rates of $<1 \mathrm{rad} /$ min were easily achieved. For vibrometer use this drift rate is much less than drifts of the experimental set-up and parts under investigation.

We have described an apparently novel multi-phase sampling and phase demodulation scheme applied to a diode-laser-sourced, fiber-delivery optical-vibrometer. The technique is applicable also to general optical sources, even when direct wavelength modulation is not possible. A noise-equivalent minimum detectable phase shift of $<50 \mu$ fringe $/ \sqrt{\mathrm{Hz}}$ was achieved with only $40 \mathrm{nW}$ received from the object surface.

\section{References}

[1] Polytec GmbH. P.O. Box 1140, D-7517 Waldbronn I, Germany.

[2] K. Weir, W.J.O. Boyle, B.T. Meggitt, A.W. Palmer and K.T.V. Grattan, J. Lighnwave Techn. 10 (1992) 700.

[3] L. Mertz, Appl. Optics 28 (1989) 1011.

[4] L. Mertz, Rev. Sci. Instrum. 62 (1991) 1356. 